\title{
Low Energy Anti-Neutrino Detection with Super-Kamiokande
}

\section{M.B. Smy ${ }^{* \dagger}$}

Author University of California, Irvine

E-mail: msmy@uci.edu

Detection of low energy anti-neutrinos in large water Cherenkov detectors via inverse beta decay reactions opens the door to the observation of the diffuse supernova neutrino background and allows high statistics measurements of the anti-neutrino flux and spectrum of distant reactors. At present, these signals are buried by backgrounds which would be greatly reduced by the observation of the produced neutrons in delayed coincidence. At present, a 200t test facility is being constructed underground near Super-Kamiokande to study the impact of the proposed addition of Gadolinium ions to the water of Super-Kamiokande in order to reliably tag neutron captures by the $8 \mathrm{MeV}$ gamma cascade emitted by the excited Gadolinium nucleus.

35th International Conference of High Energy Physics - ICHEP2010,

July 22-28, 2010

Paris France

\footnotetext{
${ }^{*}$ Speaker.

${ }^{\dagger}$ for the Super-Kamiokande Collaboration
} 

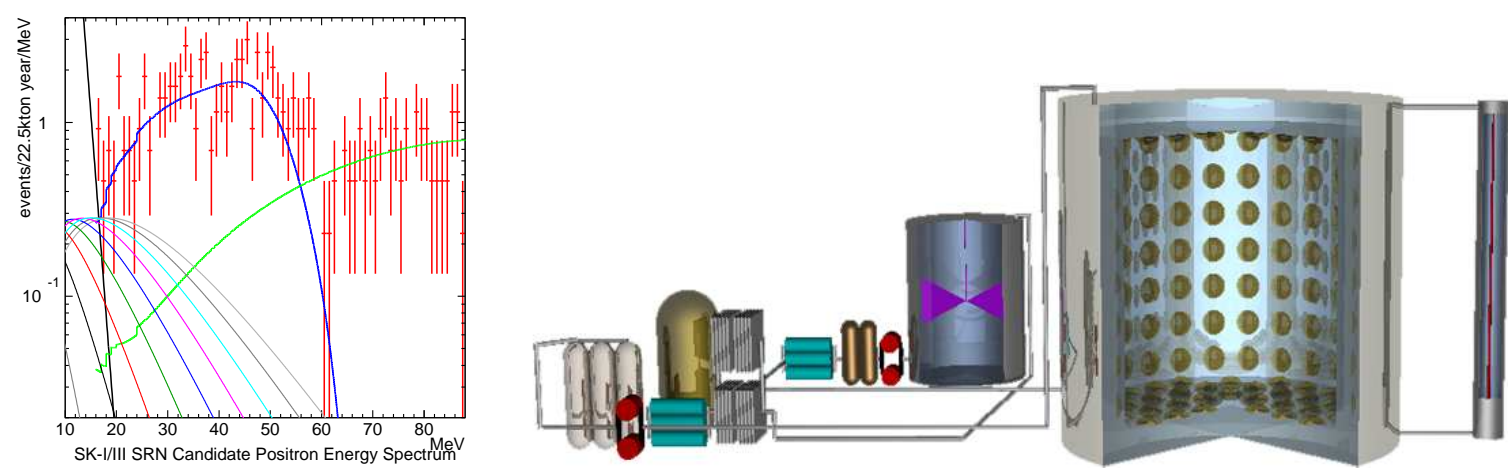

Figure 1: (a) SK-I and III Supernova neutrino candidates (red data points). The blue (green) overlay is background from sub-threshold muons (electrons) from atmospheric neutrino interactions. Predictions of supernova spectra emitted at $3 \mathrm{MeV}$ (black), $4 \mathrm{MeV}$ (red), $5 \mathrm{MeV}$ (dark green), $6 \mathrm{MeV}$ (blue), $7 \mathrm{MeV}$ (violet), $8 \mathrm{MeV}$ (cyan), as well as $2 \mathrm{MeV}, 9 \mathrm{MeV}$ and $10 \mathrm{MeV}$ (gray) are also shown. (b) Schematic of 200t test facility to evaluate the feasibility of Gd doping of Super-Kamiokande: Selective water filtration (left), Gd pre-treatment (left centre), main tank (right centre), light attenuation measurement (right).

The handful of neutrinos detected from the supernova SN1987a [1] were a astro-particle physics gold mine, but with present-size detectors only the very rare galactic neutrino burst can be detected. There also exists a constant diffuse background of neutrinos from supernovae exploding throughout the universe. Without burst, detection is more challenging and backgrounds exceed the signal over most of the energy range. Water Cherenkov detectors such as Super-Kamiokande detect either kind of supernova neutrinos predominantly via inverse-beta decay. We have searched for supernova neutrinos between 16 and $90 \mathrm{MeV}$. While the analysis is not yet finished, we produced a sample of candidates free of all backgrounds but atmospheric neutrino interactions. Between 16 and $\approx 25 \mathrm{MeV}$ we expect some sensitivity to supernova neutrinos (see Figure 1). The detection of a captured neutron in delayed coincidence will reduce backgrounds and increase the observable energy range. To aid detection of neutron captures the addition of $0.1 \%$ of Gadolinium ions was suggested [2]. This will reduce the capture time from $\approx 200 \mu \mathrm{sec}$ to $\approx 30 \mu \mathrm{sec}$ and raise the capture signal from $\approx 1.5 \mathrm{MeV}$ to $\approx 4.5 \mathrm{MeV}$ total electron energy equivalent (confirmed with a $\mathrm{GdCl}_{3}$ test source [3] suspended in Super-Kamiokande). In addition to the detection of the supernova neutrino background and determination of the effective neutrino emission spectrum, the Gadolinium-enhanced Super-Kamiokande detector could also measure the solar neutrino oscillation parameters with reactor neutrinos. In order to test the feasibility and safety of this proposal, a 200 ton detector (see Figure 1) is presently constructed next to Super-Kamiokande with selective water filtration ("bandpass" filter by particle size) and high precision measurements of the resulting water clarity (light extinction curves at seven wavelengths are measured in a $8 \mathrm{~m}$ vertical pipe). It will also check material safety, and general gadolinium handling.

\section{References}

[1] Hirata et al./Bionta et al., Phys. Rev. Lett. 58, 14901493 (1987).

[2] Beacom and Vagins, Phys. Rev. Lett., 93:171101, 2004.

[3] Watanabe et al., Astroparticle Physics 31 320-328, 2009. 